\title{
Space Robotics Research and Development Roadmaps at ESA
}

Gianfranco Visentin* * European Space Agency

\begin{abstract}
The European Space Agency (ESA) has recently completed a technology harmonization exercise for the domain of automation and robotics $(A \& R)$. ESA's harmonization exercises aim at defining a roadmap for research and development, which integrates the vision of all European players (Agencies, Industry and Academia) in the domain. The roadmap defines a series of automation and robotics end-products and the intermediate steps in research and development needed to prepare "building blocks" that can be used to "assemble" the endproducts. This paper provides the rationale for establishing the end-products, the description of their expected functionalities and their application in future missions.
\end{abstract}

\section{Introduction}

In order to compare and harmonise the many different programmes of R\&D present in Europe, a common taxonomy is needed. For this purpose ESA has established so-called technology trees. Each Technology Domain is organised in Technology Subdomains, which are further split in Technology Groups. For the technology domain of $\mathrm{A} \& \mathrm{R}$ the tree is:

\section{A. Applications and Concepts:}

Covers system aspects and innovative robotic concepts for missions.

I. Planetary Exploration: Includes novel concepts for handling/assembly of surface infrastructure elements, novel aerobot concepts, novel robot concepts for exploration (including asteroids), micro and nano rover concepts and swarms.

II. Orbital Systems: Includes automation of orbital infrastructure, or non-cooperative satellites, satellite design for robotic servicing, compound operations

原稿受付 2009 年 3 月 9 日

キーワード : R\&D Planning, Space Robotics

*P.O. box 299, 2200AG, Noordwijk, The Netherlands of arms on free-flying platforms, assembly and servicing of space structures in orbit, multi-robot cooperation.

\section{B. Automation \& Robotics Systems:}

Covers the detailed definition of robotic systems and subsystems, including technology developments dedicated to specific applications.

I. Manipulation systems: Includes robot arms, endeffectors and tools.

II. Mobility Systems: Includes Rovers, Aerobots, underground and underwater explorers.

III. Payload Automation Systems: It covers all automation aspects of space laboratories.

C. Automation \& Robotics components and Technologies:

Includes general purpose and specific A\&R components and methods.

I. Perception: Includes sensors and sensing methods (e.g. computer vision), which allow robots to perceive their environment and the state of the process they control.

II. Control, Autonomy and Intelligence: It covers methods and means that allow robot systems to perform perception processing, understanding of the operating environment, motion planning and control, attention allocation, anticipation, activity planning, and reasoning about their own state and the state of other agents.

III. Motion and Actuation: It covers the means that allow a robot to physically interact with its environment (e.g. limbs, joints, chassis, wheel units, balloon envelopes, propulsion units).

IV. Robot-User Interfacing: Includes commanding and programming means (e.g.immersive systems, haptic devices) and methods that allow users to interact with an automation and robotics system. Includes Teleoperation, Telepresence, Telescience. 
V. Robot Ground Testing: Includes tools, methods and facilities that allow on-ground characterization and verification of $A \& R$ systems.

\section{Technology Trends}

\subsection{Applications and Concepts: Planetary Exploration}

On the base of terrestrial technology trends on the base of possible planetary exploration missions it can be argued that robotics means will take the form of:

-Robot assistants: to support crew operations on the Moon. They will likely take the form of "centaur" robots, i.e. a humanoid upper-body with 6 - wheeled or 6-legged lower-body.

- Robot agents for the exploration of poorly reachable scientific sites (e.g. Moon poles craters).

They will likely take the form of climbing/rappelling systems (legged or wheeled) equipped with instrumented robot arms.

- Robot explorers for returning samples from remote celestial bodies (e.g. Mars, Deimos, Asteroids). They will likely take the form of vehicles (rovers for Mars and hoppers for the low gravity environments) with sophisticated sampling and sample-preparation tools.

- Robot explorers for carrying scientific instruments in the atmosphere and into the underground of celestial bodies. They will take the form of aerobots (balloons, gliders) and smart moles.

\subsection{Applications and Concepts: OrbitalSys- tems}

The trend for orbital systems is towards:

- Robot assistants for large orbital infrastructure, to assist crew in assembly and maintenance tasks. They will likely maintain the form of the present Eurobot system

- Robot agents to maintain and service GEO platforms. They will likely take the form of the present Eurobot system with the additions of a relocation system (a deployable scaffold or large robot arm)

- Robot explorers for opportunistic science from orbit. These systems will be just custom-built conventional spacecrafts with powerful autonomous software (derived from the one currently being developed for autonomous rovers) capable to react to environmental changes/events and trigger acquisition of scientific data.

\section{Mission Needs}

Considering the trend in space exploration and utilisation, the following application scenarios appear very likely:

1. Maintenance and operation of the ISS: the ISS represents a huge investment for Europe. While there is evidently European reluctance to further invest in the ISS, it appears that its use, if done in the most economic way, is well justified and accepted. Robotics will allow efficient use of the ISS with negligible investment. Furthermore there is no longer opposition of astronauts against robots.

\section{Maintenance of Geostationary orbital infras-} tructure: there is a mounting number of missions proposed for servicing GEO satellites in orbit. DARPA alone has already two missions (Orbital Express and SUMO) in the pipeline, while ESA and DLR have long worked on the subject with very promising results. It is foreseeable that a technology demonstration mission will materialize in the reference period.

3. Participation to Moon exploration activities: NASA has committed to the return to the Moon. ESA and ASI have investigated what could be their respective contribution to an International manned mission to the Moon. Both investigations conclude that robotics elements are a convenient contribution. Finally a particular interest at ESA and also at some National Agencies is the exploration of Lunar crater poles. ESA is currently studying a mission to follow ExoMars before the end of the 2010 decade. One of the two candidate missions aims at sample return from a crater in the Moon's south pole.

4. Sample return from the Martian system: NASA and ESA have studied possible missions for returning soil samples from Mars or Deimos. A joint mission has been postponed to time to be decided. However both ESA and NASA have started to work on technology for such mission.

5. Opportunistic science mission: The possibility exists that small/smart robotics means could be installed within an existing scientific spacecraft or delivered to the surface of a planet as additional payload. For this reason ESA has developed in the past the Nanokhod microrover, technology for microprobes, technology for a small motor-glider and technology for balloon smart-science collection. 


\section{Roadmaps to the End-Products}

For the R\&D programmes that focus on TRL up to 4, the development approach chosen, was based on the construction of technology building blocks that can be re-used for different robotic end-products. The 2 derived roadmaps show end-products and the building blocks that enable the making of the endproducts. The roadmap only shows building blocks that represent critical missing technology to be developed (i.e. an endproduct is not formed only by the building blocks shown in the roadmap). Also the completion of all building blocks in a roadmap does not mean that the endproducts are ready: availability of all missing technology is just the pre-requisite to start the development of the end-product. Finally, the critical missing technology in the roadmaps has been chosen with the aim to be affordable: it can be accommodated in a budget based on a benign extrapolation of the ESA R\&D expenditure in $A \& R$ in the last ten years. More technology would be needed to make the end-products. However as resulted in the previous harmonization exercise it is important to identify clear priorities fitting with a reasonable budget level.
4.1 Roadmap 1: GEO robotic agent, ISS robotic crew assistant, planetary robotic agent, planetary robotic crew assistant

This roadmap (see Fig. 1) aims in first instance to a GEO robotic agent (to be used for teleoperated dexterous manipulation on orbital infrastructure) and in second instance to a semi-autonomous robotic crew assistant. Much of the same technology, together with additional building blocks can also be used to prepare for a Lunar robotic crew assistant (to provide assistance to astronauts on the lunar surface) and a Lunar robotic agent (to be operated from Earth in the exploration of Lunar pole craters).

\section{2 Roadmap 2: Sample Return Robotics, Orbital Robotic Explorer, Instrument carrying Planetary explorers}

This roadmap (not shown for reasons of space) will use some building blocks made ready by the previous roadmap. End products are technology to enable a more sophisticated Mars sample return mission (featuring a stationary lander and a sampling rover), an adaptation of the Autonomous Robot controller to support opportunistic science in spacecrafts, and finally a continuation of the several ESA sponsored technologies that allow "Instrument Carrying Explorers" to operate

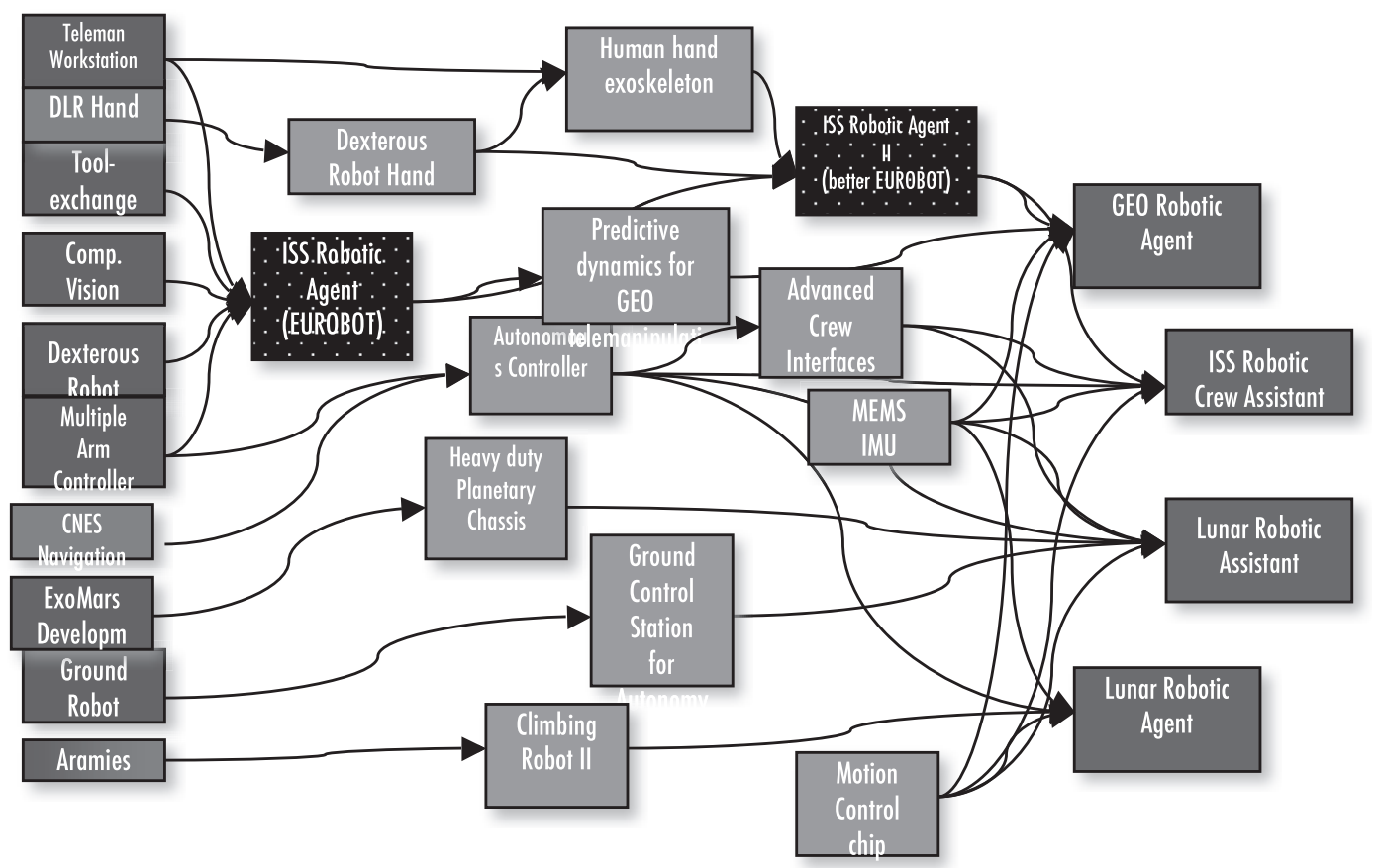

Fig. 1 Roadmap 1; for clarity only main building blocks are shown 
in the atmosphere or in the underground of planets.

\section{Conclusions}

There is universal agreement that the A\&R will play an essential role in Space Science and Exploration as well as in Space Exploitation (space power production, space manufacturing, space mining) and in the development of innovative commercial space applications (satellite servicing, large space infrastructure). These missions will see:

- Robot Assistants: helping Astronauts to perform their task quicker, safer, with higher quality and more economically.

- Robot Agents: working into hostile and dangerous areas and acting in place of humans to perform assembly, maintenance and production tasks in teleoperated or semi-autonomous way.

- Robot Explorers: venturing in the hostile and remote planetary environments for exploration and science. Coping with and taking advantage of the peculiar environmental conditions to achieve long duration and extensive range missions. ESA, in harmony with all European players in A\&R has devised a plan for developing basic technology to enable the above robots.
Acknowledgments The author wishes to thank the members of the ESA Technology Harmonisation Group (THAG), without the kind cooperation of which, the material that forms the basis of this paper would not have been produced.

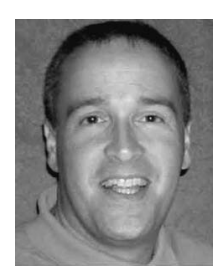

\section{Gianfranco Visentin}

Mr. Gianfranco Visentin has been with the European Space Agency (ESA) for the last 18 years. He had previously worked as control engineer on aircraft flight software and active car body attitude control. Since his beginning at ESA he has been with the Automation and Robotics (A\&R) group working in support of ESA robotics projects and in Research and Development (R\&D). In supporting ESA projects he has participated to the development of the European Robot Arm (ERA), the Columbus Microgravity Facilities, the EUROBOT system (of which he was the initiator) and the ExoMars project. His R\&D efforts have covered the whole spectrum of technologies needed for space A\&R including: conventional robotics platforms (rovers, robot arms), alternative robotic platforms (moles, aerobots, walking robots), robot autonomy, teleoperation and remote control (robot programming stations, exoskeletons), perception (computer vision) and subsystems (robot joints, controllers). Since 2002 he leads the A\&R group. In his current post Mr. Visentin is ESA's responsible of the technology domain for Automation and Robotics, role that entails the preparation of ESA's R\&D strategy for the field and coordination with other European research organizations. 